\title{
Dedicatoria al doctor Jesús Kumate Rodríguez
}

$\mathrm{E}^{\mathrm{l}}$ grupo editorial de Salud Pública de México dedica este número especial sobre los estudios seroepidemiológicos de la Encuesta Nacional de Salud 2000 al doctor Jesús Kumate. Este merecido reconocimiento a una de las figuras médicas mexicanas más importantes del último medio siglo distingue así sus enormes contribuciones en los campos de la infectología clínica, la investigación biomédica y la salud pública. En efecto, Jesús Kumate fue de los primeros en reconocer la importancia de las encuestas de salud en general, y de las seroepidemiológicas en particular. ¿Cómo conocer la verdadera prevalencia de enfermedades infecciosas en la población general, en ausencia de un sistema sofisticado de vigilancia epidemiológica? Sus clásicos libros -publicados originalmente en los años setenta- "Manual de infectología" y "La salud de los mexicanos" están llenos de recomendaciones terapéuticas o políticas públicas sustentadas en la mayor evidencia científica en su momento, así como del señalamiento atinado de la necesidad de contar con un mejor sistema de información en salud.

Fue así que nacieron las primeras encuestas seroepidemiológicas en nuestro país. En el Instituto Mexicano del Seguro Social (IMSS) se llevó a cabo la primera encuesta recogiendo sueros entre los derechohabientes. Pero fue hasta el año de 1987 cuando se levantó la primera encuesta seroepidemiológica verdaderamente representativa de todos los habitantes de nuestro país, bajo el ojo observador del entonces subsecretario Jesús Kumate.

Los resultados de las encuestas de salud en México han permitido tomar decisiones basadas en sólida información científica para generar políticas públicas. Por poner un ejemplo de la importancia de lo anterior, basta citar la complacencia que existía en las autoridades sanitarias, antes de las encuestas de salud, al contar con la falsa seguridad de tener altas coberturas de vacunación -de acuerdo con los registros administrativos de vacunas aplicadas- en los años ochenta antes de la gravísima epidemia de sarampión de finales de esa década. Esta tragedia detonó la creación del Programa de Vacunación Universal, ideado por el doctor Kumate, tomando como base la información sobre las verdaderas coberturas en la población infantil en una encuesta epidemiológica en cerca de 250000 hogares representativos a nivel nacional.

Son tan extensas las contribuciones del doctor Kumate a la ciencia y a la salud en México que sería imposible tratar de abarcarlas todas en esta dedicatoria. A manera de apretado resumen, se listan a continuación algunos de sus cargos y honores recibidos en su productiva vida profesional y académica.

Editor del Boletín Médico del Hospital Infantil de México (1956-1957); jefe de la División de Investigación del Hospital Infantil de México (1960-1979); director del Hospital Infantil de México (1979-1980); coordinador de los Institutos Nacionales de Salud SSA (1983-1985); subsecretario de Servicios de Salud SSA (1985-1988); secretario de Salud (1988-1994); presidente del Consejo Ejecutivo de la Organización Mundial de la Salud, Ginebra(1994-1995); jefe de la Unidad de Investigación Médica en Bioquímica, CMN SXXI, IMSS (1981-1985 y 1995-2003); presidente ejecutivo de la Fundación IMSS, A.C.(2003 -); presidente del Patronato Nacional de Centros de Integración Juvenil (2000-); Patrono Fundación Gonzalo Río Arronte (1994-2006).

El doctor Kumate es miembro de El Colegio Nacional y ex presidente de la Academia Nacional de Medicina de México, así como integrante emérito del Sistema Nacional de Investigadores y doctor honoris causa de dos universidades.

Ha publicado 367 artículos en revistas y libros de divulgación, revisión e investigación, y 19 libros. En varias de sus publicaciones se presentan los resultados de encuestas seroepidemiológicas dirigidas por él. Los trabajos del doctor Kumate se orientaron inicialmente a las enfermedades infecciosas, la inmunología y las vacunas, pero en fechas recientes la diabetes mellitus ha sido uno de sus temas prioritarios de investigación. 
Entre otros muchos premios y distinciones, recibió la condecoración oficial de Caballero de la Legión de Honor de Francia. La Secretaría de la Defensa Nacional lo reconoció por Servicios Distinguidos. Recibió el Collar de la Orden de Rodolfo Robles de Guatemala, la Banda del Tesoro Sagrado de Japón y la Orden al Mérito en grado oficial del gobierno de Italia. El mayor y más reciente premio fue la Medalla Belisario Domínguez del Senado de la República, que se le otorgó en 2006.

Sin la inspiración de sus trabajos de investigación y su apoyo como funcionario en las encuestas nacionales de salud, los trabajos que se presentan en este suplemento de Salud Pública de México no hubieran podido realizarse.

Por las razones expuestas anteriormente, resulta apropiado dedicar este conjunto de artículos al maestro, investigador y funcionario Jesús Kumate. 